\title{
Carrier Ethernet "label" scalability
}

Luis Fernando Caro, Jose Luis Marzo

University of Girona

Universitat de Girona

Dimitri Papadimitriou

Alcatel-Lucent

Alcatel-Lucent 
Outline

- Introduction

- Ethernet VLAN-Label Switching (ELS)

- Provider Backbone Bridge - Traffic Engineering (PBB-TE)

- The Label Space Reduction Problem

- Related Work

- Simulation results

- Conclusions 
Introduction

- Ethernet has been increasingly attracting service providers and the telecommunication community as the technology for Transport/Aggregation networks.

- Some of the advantages of Ethernet are:

- Interfaces that go from $10 \mathrm{Mb} / \mathrm{s}$ to $10 \mathrm{~Gb} / \mathrm{s}(100 \mathrm{~Gb} / \mathrm{s}$ for 2010$)$

- CAPEX reduction, more bandwidth for less price

- Flexibility

- Increment on Demand for Ethernet Services (E-LINE,E-Tree, E-LAN) over Transport/Aggregation networks. 
Introduction

- However, when considering bridged Ethernet networks in a carrier environment there are several short comings:

- Traffic isolation

- Ethernet MAC address learning

- Lack of traffic engineering capabilities

- Network recovery

- Extending the reach of Ethernet technology toward aggregation networks requires enhancing its properties in order to become a carrier grade technology.

- Two techniques are studied: Provider Backbone Bridge - Traffic Engineering (PBB-TE) and Ethernet VLAN-Label Switching (ELS). 
Ethernet VLAN-Label Switching (ELS)

- ELS uses the Provider Bridges PB (802.1ad) standard and encodes the label in the S-VID tag field.

- GMPLS can be used at the control plane.

- Intermediate Nodes can perform label swapping and the domain of the labels is assumed to be link local. Label Stacking is not allowed.
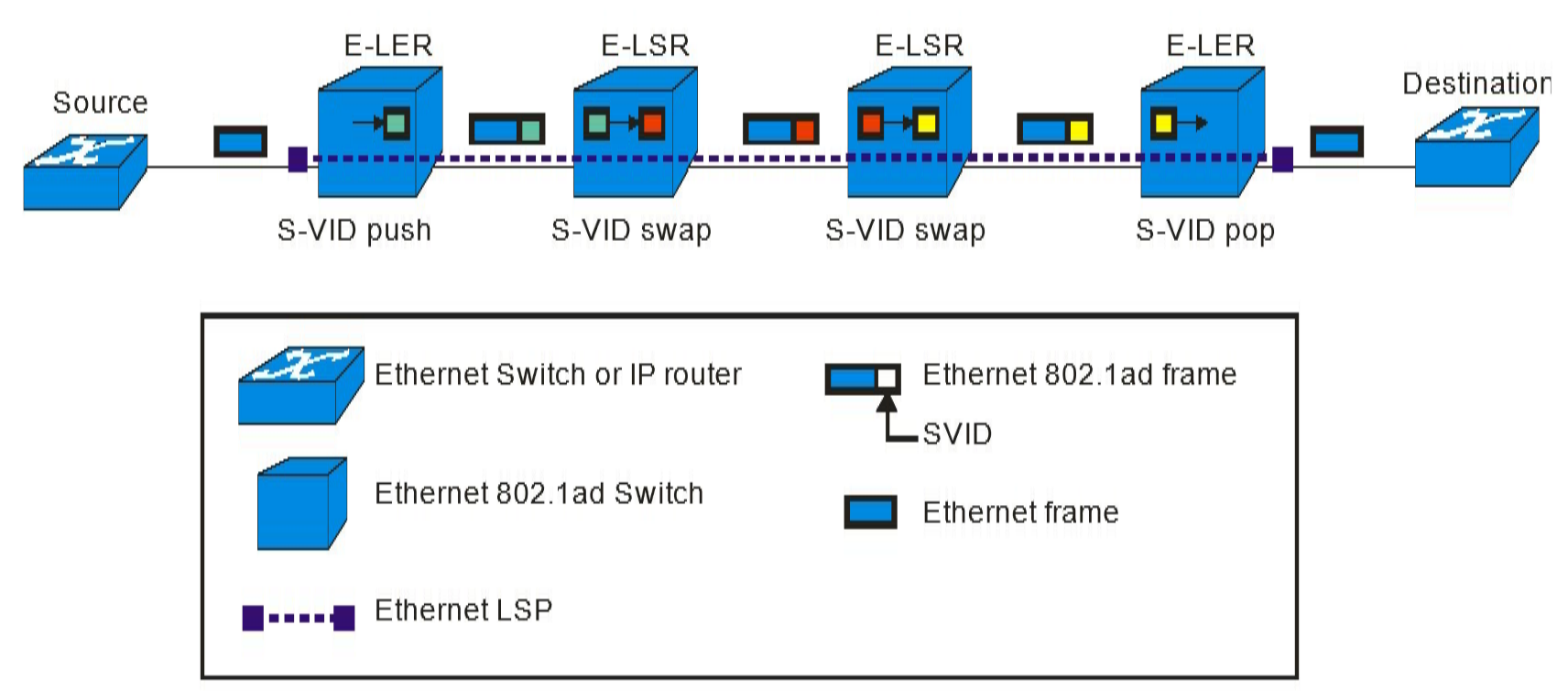


\section{Provider Backbone Bridge - Traffic Engineering (PBB-TE)}

- PBB-TE uses the Provider Backbone Bridges PBB (IEEE 802.1ah) standard and encodes the label in the S-VID tag field.

- It is being standardized as IEEE 802.1ay

- GMPLS can be used at the control plane

- Forwarding is done based on VLANID +Destination MAC address. Intermediate Nodes cannot perform label swapping or stacking and the domain of the labels is assumed to be per destination.

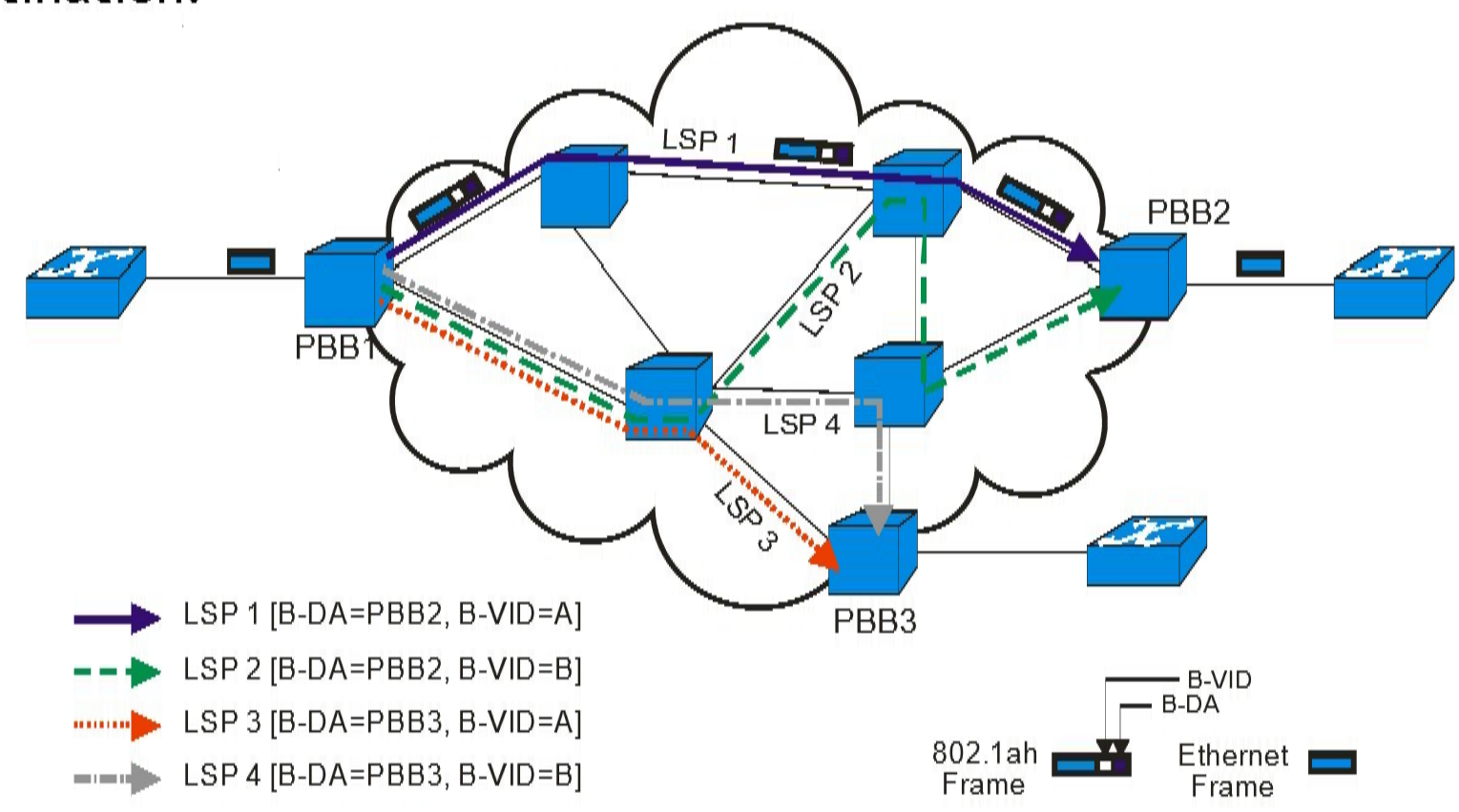




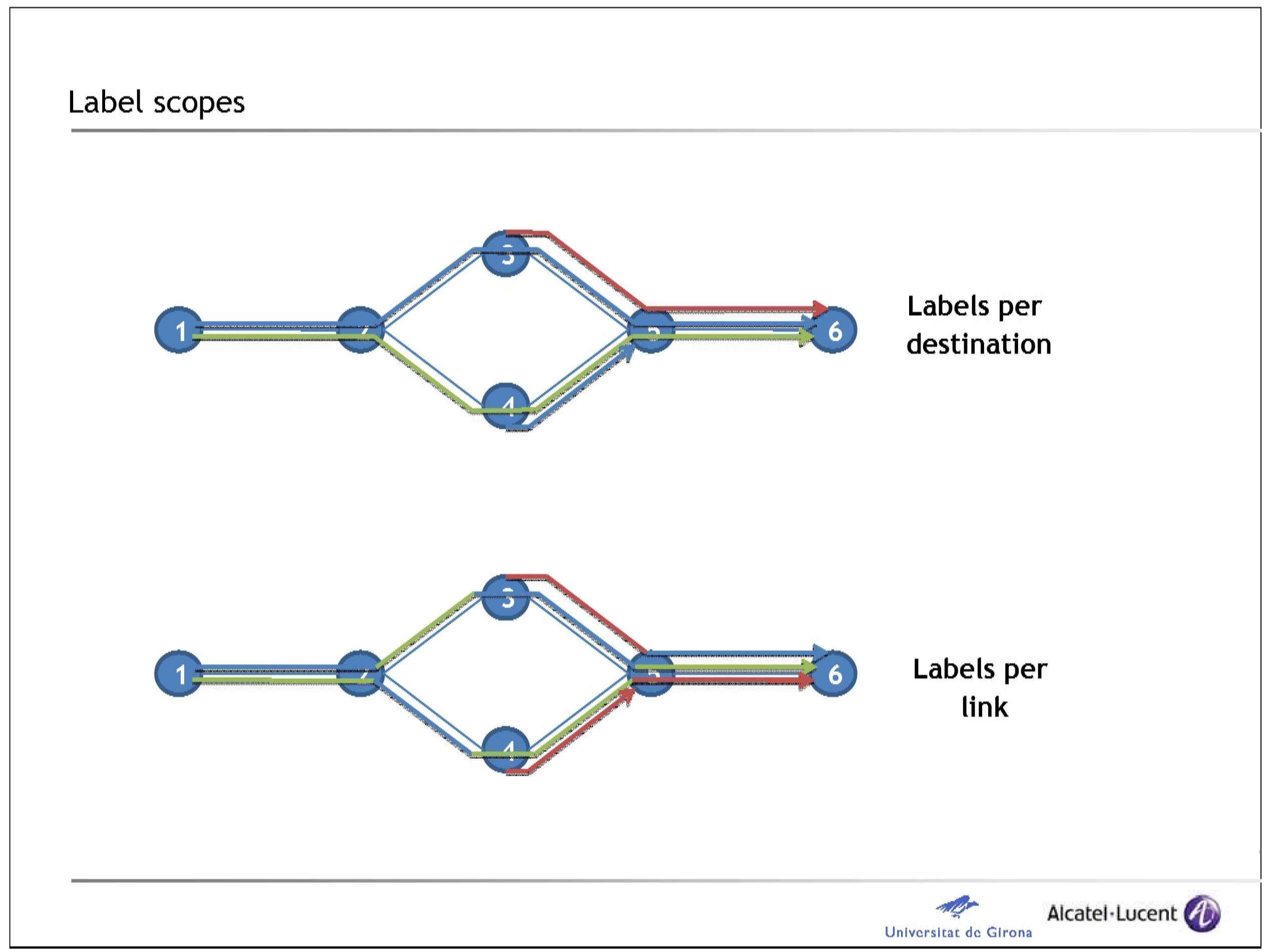

Authorized licensed use limited to: UNIVERSITAT DE GIRONA. Downloaded on May 8, 2009 at 07:03 from IEEE Xplore. Restrictions apply. 
The Label Space Reduction Problem

- In the case of ELS are encoded using the VLAN ID field of the Ethernet frame. Therefore the maximum number of LSP that can be forwarded through an interface is $4.096\left(2^{\wedge} 12\right)$ LSP.

- In the case of PBB-TE, labels are globally unique and encoded on both B-VID and B-DA fields. Therefore a maximum of 4096 LSPs per destination MAC address can be created.

- In MPLS the maximum is $1.048 .576\left(2^{\wedge} 20\right)$ per interface without considering stacking.

- The label space problem in can be defined as: given a routing scenario, how can the Ethernet LSPs be set up in the network so that corresponding bandwidth requests are not rejected due to scarcity of labels when the unreserved bandwidth in the corresponding links has not reached 0.

- Two techniques can be used in label switching architectures to assign the same label to different LSPs. Label merging and label stacking. 


\section{Label Merging Technique}

- Label merging can be used in label switched networks where nodes are capable of performing label swapping.
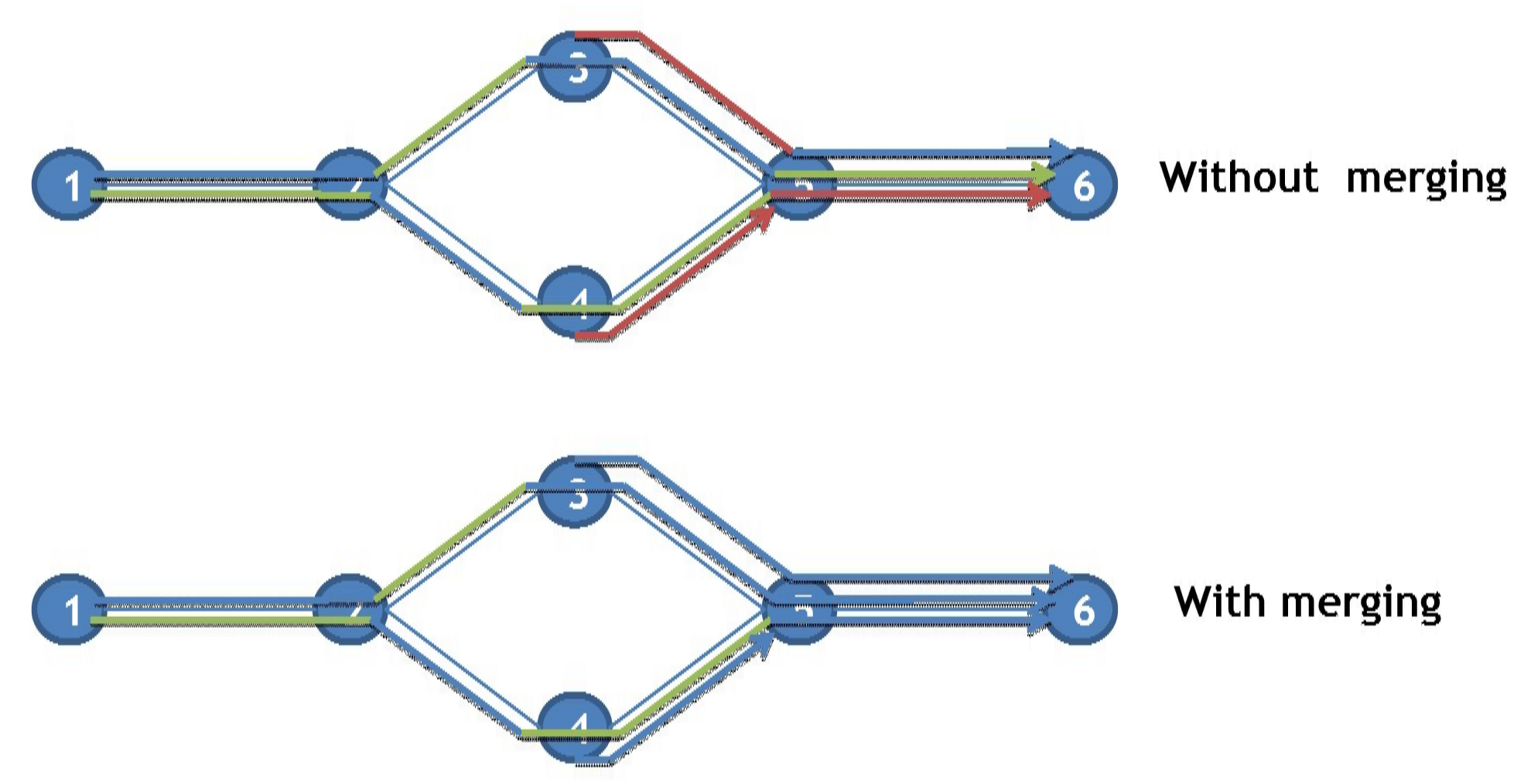


\section{Label Reutilization Technique}

- Label reutilization consists of assigning the same label to two or more LSP that are completely link disjoint.

- It has been shown that optimal label assignment is NP-Complete
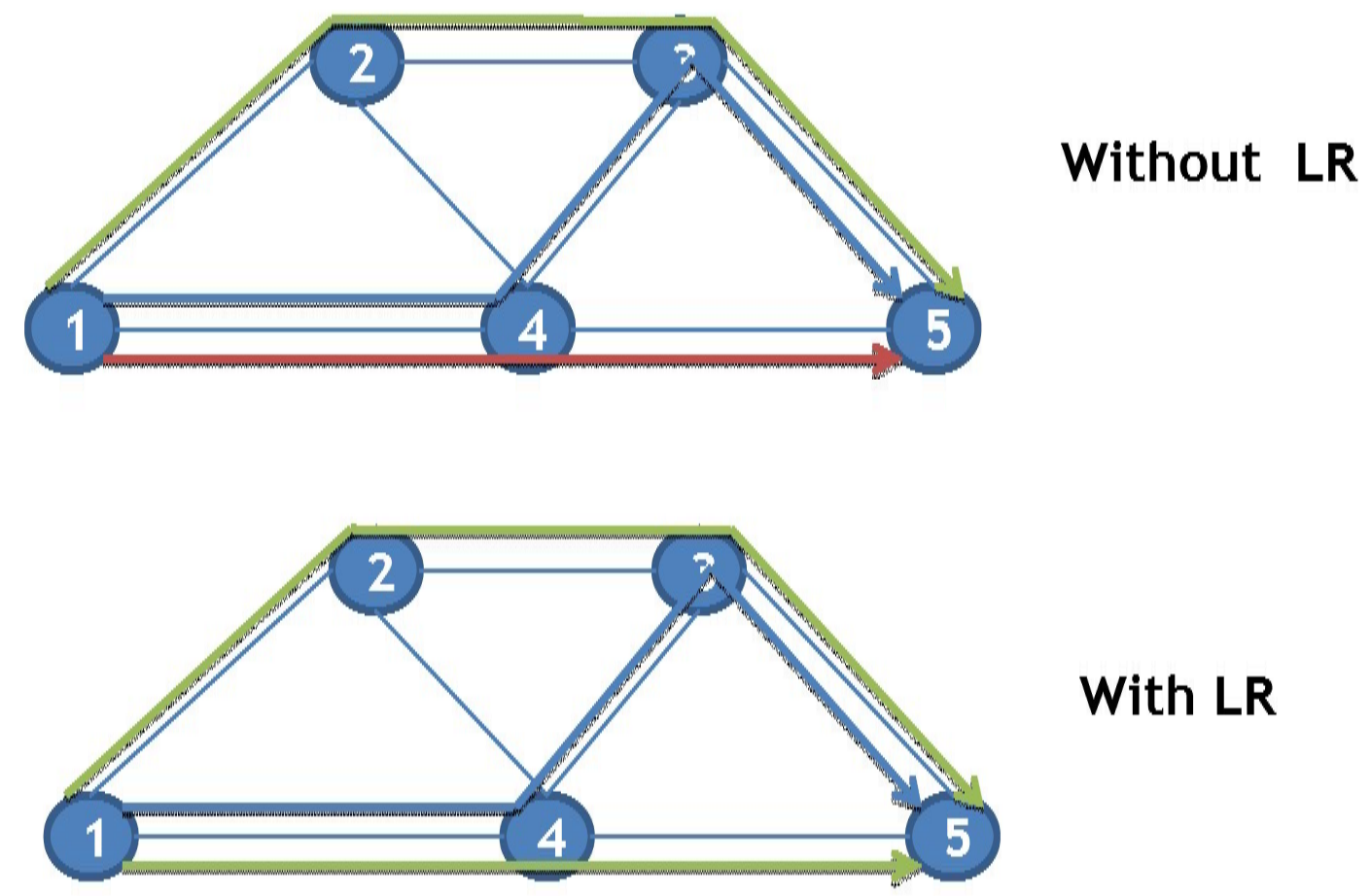
- Label space reduction has been studied in MPLS with the objective of simplifying network management, reducing Operational Expenditure (OPEX) and LSP state message processing.

- Two scenarios have been studied for MPLS:

- Offline routing scenario: [1] proposes an offline routing algorithm that bounds the forwarding table sizes to $(M+N)$.

- LSPs routes given scenario: The goal is to determine the operations performed at each node (swapping or stacking) so that the total number of labels used in the network is minimized. [2] shows that an optimal solution for label merging can found in polynomial time. Other related work uses stacking or merging.

- The difference with previous studies is that the main goal of this work is to analyse the limitations of using a 12 bit label without stacking.

[1] D. Applegate and M. Thorup. Load optimal MPLS routing with N+ M labels. In INFOCOM 2003.

[2] Fernando Solano, Ramon Fabregat, and Jose Marzo. On optimal computation of MPLS label binding for multipointto-point connections. IEEE Trans. Commun. 2007. 
- The existing routing algorithms considered in these simulations are the Shortest Path First (SPF) (cost, hop count), the Constraint Shortest Path First (CSPF) (delay, unused capacity, hop count), and the minimum interference routing algorithm (MIRA).

- In order to effectively obtain meaningful results three topologies of different sizes are considered: Cost266(37), Germany(50), and Exodus(79). Their link capacity is set to $10 \mathrm{~Gb} / \mathrm{s}$ and for each topology two sets of bandwidth requests serve as input:

- An Homogeneous request set: where each bandwidth request is set to $1 \mathrm{Mb}$

- An Heterogeneous request set: where the bandwidth of each request is selected from $1 \mathrm{Mb}, 2 \mathrm{Mb}, 10 \mathrm{Mb}, 20 \mathrm{Mb}$.

- The algorithms performance is evaluated in terms of the sum of the accommodated bandwidth of all the established LSPs in the network (Throughput). 
Simulation results : Homogeneous request set

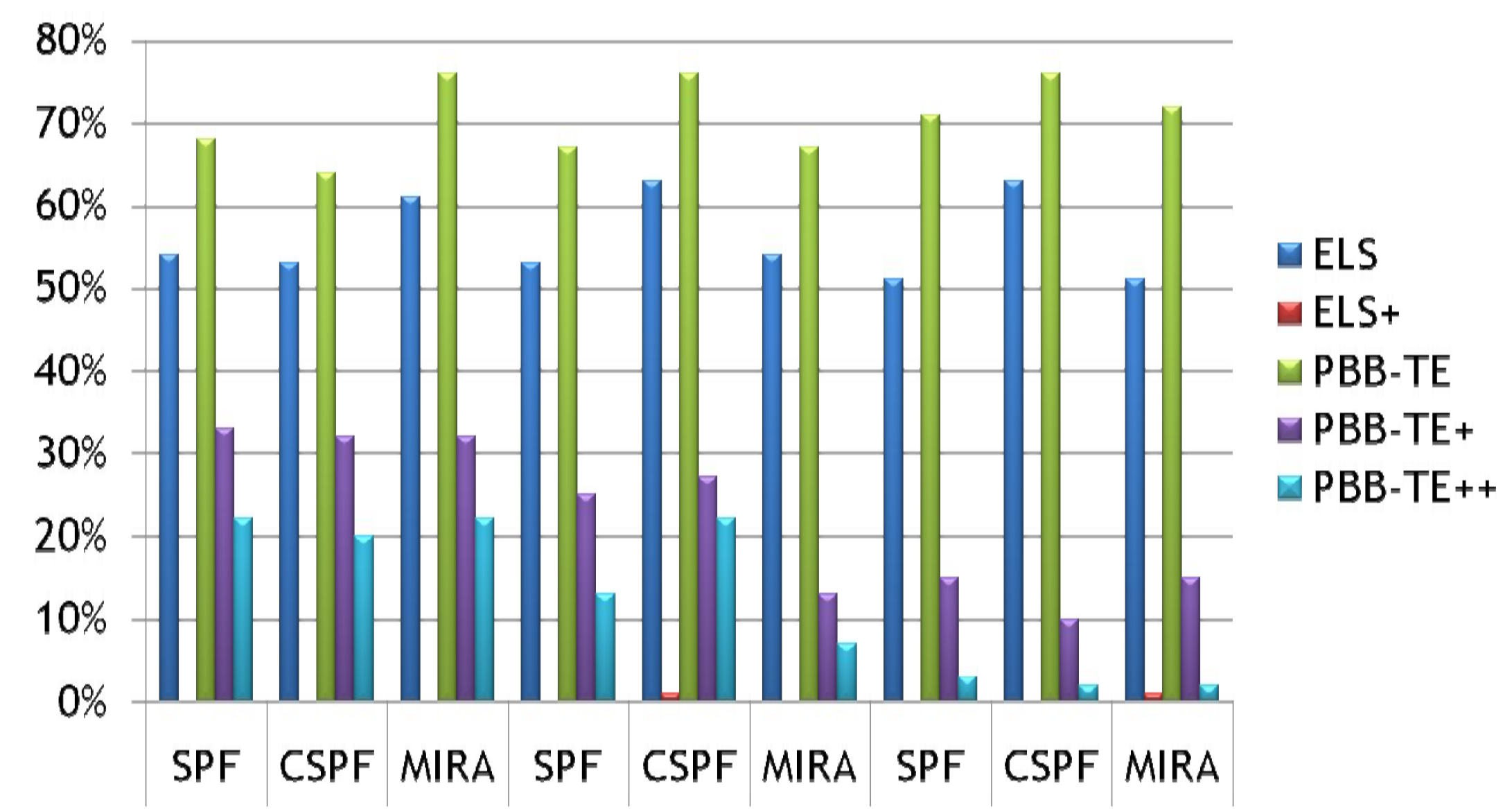

Alcatel-Lucent (4) 
Simulation results : Heterogeneous request set

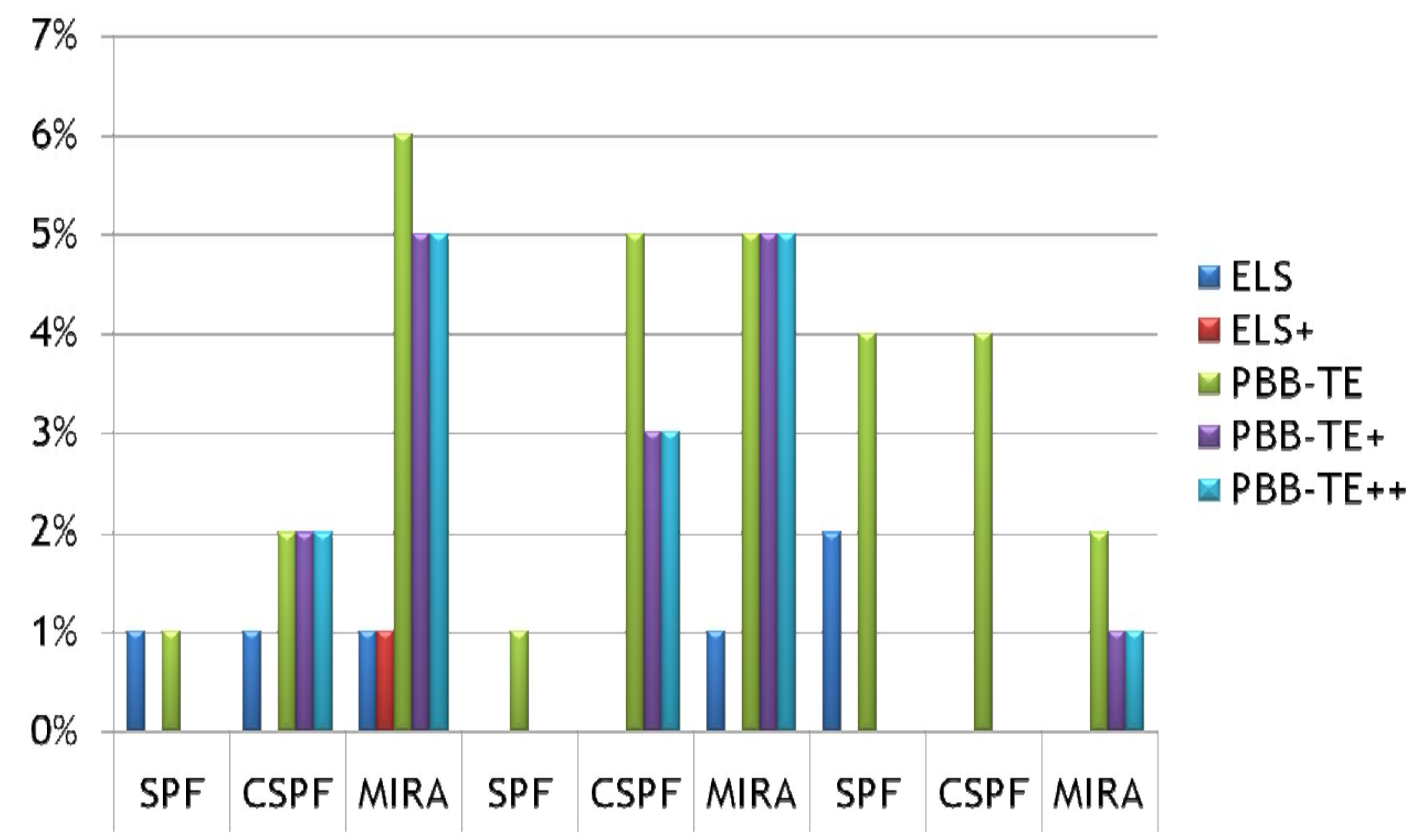




\section{Conclusions}

- Results for the online routing scenario show that when demands of $1 \mathrm{M}$ are considered, a 12 bit label can result in performance degradation when no label reduction techniques are used. However, applying label merging and reutilization allows performance to be maintained in terms of accommodated traffic load.

- For labels per destination there was a difference of at most $13 \%$ of decrease in throughput among label assignment schemes.

- Both Label merging and reutilization have to be taken into account in the development of carrier Ethernet technologies.

- Future work includes a topology dependency study of label space usage. 